\title{
Establishing Practice Risk Management and Outcomes Claims for Medical Marijuana Dispensaries: Questions Legislators Should Ask
}

Paul C Langley, PhD

Adjunct Professor, College of Pharmacy, University of Minnesota

\begin{abstract}
In a previous commentary in INNOVATIONS in Pharmacy, the case was made that a major oversight in approving the establishment of medical marijuana programs through commercially and not-for-profit operated dispensaries is the failure to put in place standards for the monitoring and reporting of outcomes. It was pointed out that the evidence base is limited for the range of dosing options, administrative routes and conditions treated. The concern is that the ease that patients have in obtaining medical marijuana certification in many states means that a medical marijuana program is, in effect, little different from a recreational program. Dispensaries understandably focus on sales and returns to investors with scant attention given to tracking and reporting outcomes across the range of conditions and symptoms presented. While this no doubt appeals to investors in reducing administration costs, it makes it virtually impossible to deliver the appropriate and coordinated level of care that patients should expect if a medical marijuana dispensary is to meet it responsibilities in its duty of care. This places dispensaries at malpractice risk. Given this, this commentary focuses on the questions that legislators should ask in licensing medical marijuana dispensaries to ensure they meet a defensible duty of care to their patients.
\end{abstract}

Keywords: Medical marijuana, dispensary registry, quality assessment questions, legislative standards, duty of care

\section{INTRODUCTION}

Do medical marijuana dispensaries have a duty of care to their patients? If we accept the proposition that an approved medical marijuana dispensary is a healthcare provider, then it is under a reasonable obligation to ensure that the focus is on achieving the best possible clinical outcomes while at the same time endeavoring to minimize patient harms. The notion of duty of care also implies that, in the delivery of care, the provider is obliged to attempt to ensure than the care is appropriate, that it is monitored and reviewed against targets set for the patient, and that the outcomes achieved are consistent with best clinical practice.

The problem with botanical cannabis is that the evidence base for establishing the appropriate dosing regimen and administrative form is limited or simply non-existent for the range of approved conditions. The fact is that the dispensing of botanical cannabis for the range of disease states and conditions authorized under medical marijuana legislation occurs in what is best described as a fragmented evidence environment. This should give legislators pause. If there is the potential for harm, given the range of clinical and comorbid conditions presented, for example by older patients, then as argued in the previous commentary there needs to be a care management framework ${ }^{1}$. The primary care provider or care manager should be linked to the dispensary and provided with regular real-time reports of the response to therapy.

Corresponding author: Paul C Langley, PhD

Adjunct Professor

College of Pharmacy University of Minnesota

Director, Maimon Research LLC

5061 North Apache Hills Trail, Tucson, AZ 85750

Tel: 520-577-0436; Email: langley@maimonresearch.com
Unfortunately, from the limited evidence available, this appears to be almost entirely absent in those states that have legislated for medical marijuana. There is no requirement for feedback to primary care providers or even specialist clinics in the case, for example, of severe and chronic pain. All too often, there is no necessary requirement that the PCP or specialist be involved in the certification process with certification provided by independent operators who may have only a limited knowledge of the patient's medical history and no incentive to track how the patient responds to therapy; apart from a cursory annual re-certification.

The purpose of this commentary is to make the case that a dispensary should meet minimum quality standards in monitoring and evaluating response to therapy. This has two benefits: (i) it demonstrates to patients, providers and legislators that the dispensary is committed to a duty of care in the provision of botanical marijuana and (ii) that the dispensary is committed to make a contribution through its observational platform to a better understanding of the evidence base for the contribution of botanical cannabis in the delivery of healthcare. The patient, after all, is the principal beneficiary and is entitled to the appropriate standard of care.

\section{EVIDENCE STANDARDS AND REQUIREMENTS}

In the previous commentary the limited and fragmentary nature of the evidence base for botanical cannabis was noted. More recent reviews reinforce this conclusion. Of particular interest is the December 2018 report of the European Monitoring Centre for Drugs and Drug Addiction on the medical use of cannabis and cannabinoids ${ }^{2}$. The report points out that access to medicinal marijuana, with the few exceptions of prescription cannabinoids in epilepsy, has occurred without legislators or regulatory agencies requiring evidence standards that would apply in marketing authorization for new medicinal 
products. More to the point is the absence of a strong evidence base for a range of conditions for which claims have been made, particularly against potentially more effective compounds. In the case of chronic non-cancer pain, including neuropathic pain, which is by far the most common condition treated, the evidence is considered moderate with a small but statistically significant effect for botanical cannabis compared to placebo. The 'strongest' evidence was for neuropathic pain with inhaled vaporized botanical cannabis. From a Bayesian meta-analysis of five randomized controlled trials (RCTs) $(n=178)$ the benefit over placebo was a $30 \%$ reduction in pain at 2 weeks ${ }^{3}$. A Cochrane review of neuropathic pain, again against placebo, found a modest gain from 16 studies ( $n=1750$ ) with $21 \%$ as opposed to $17 \%$ achieving a $50 \%$ reduction in pain for $2-26$ weeks. A $30 \%$ reduction is pain was achieved by $39 \%$ of patients (cf. 33\% placebo) ${ }^{4}$. Finally, in a review by Stockings et al, of chronic non-cancer pain, 91 studies from 47 randomized clinical trials ( $n=9958$ ), the corresponding percentages for patients achieving a $30 \%$ reduction in pain was $29 \%$ for those with cannabinoids compared to $26 \%$ with placebo ${ }^{5}$. While this last difference was statistically significant it points to two factors that need to be considered in legislating medical marijuana: (i) in a placebo comparison the results are mixed, with more favorable results for neuropathic pain, although across all noncancer pain patients the average gain in pain reduction is modest; and (ii) that the majority of patients in these trials reported either no gain or less than a $30 \%$ reduction in pain over the course of the study.

There is no halfway house. Either there is a commitment to a robust program of RCTs and observational studies, with dispensaries having a potentially major contribution to make in the latter area, or we continue as we are at present with a fragmented and limited evidence base. Unfortunately, at present, only one state with a medical marijuana program, Minnesota, has reported on patient experience with botanical cannabis. Following the addition of intractable pain as a condition to the medical cannabis program in August 2016, a study was undertaken of enrollee experience during the first 5 months of treatment (to December 2016). The healthcare practitioner assessed pain status with intractable pain defined as 'pain whose cause cannot be removed and, according to generally accepted medical practice, the full range of pain management modalities appropriate for this patient has been used without adequate result or with intolerable side effects'. The study involved 268 health care practitioners registered with the state medical cannabis program who certified the 2,245 patients covered in the report for intractable pain. The report draws on data assembled at each time cannabis was purchased at a dispensary together with surveys of both providers and patients.

While it is not the intent here to describe the structure and findings from the Minnesota study in detail, there are a number of points to emphasize. In the first place, criticisms from an evidence grade perspective can be made of this and similar studies, in this respect the absence of a control group and the possibility of a significant placebo effect, reinforced by the form of cannabis consumed, on the outcomes reported by patients. Even so, the Minnesota model demonstrates the feasibility of establishing dispensary specific databases or registries to monitor the impact of botanical cannabis. Second, the Minnesota study points to the importance of data collection on patient characteristics and medical cannabis purchasing at each visit over the course of treatment with products classified by their delta-9 tetrahydrocannabinol (THC) to cannabidiol (CBD) ratio. Indeed, identifying these ratios points to a concern: the fact that very high THC products accounted for some $57 \%$ of product purchases, with only $5 \%$ of products classified as high or very high CBD. Third, the Minnesota survey points to the importance of linking patient responses to providers and, if possible, administering a parallel set of questions to providers (e.g., opioid utilization after initiation of botanical cannabis). Fourth, as the Minnesota study illustrates, it is imperative, from the perspective of establishing an evidence base, that it should be one that can link to claims from RCTs and observational instruments. This means a commitment to utilizing validated studies to establish patient status and change over the course of treatment. Otherwise we run into problems in establishing credible claim indices, evaluating claims and replicating these claims in other treating environments. The Minnesota study, for example, utilizes the validated 3 -item PEG instrument ${ }^{6}$. This captures three dimensions of pain experience: average pain last week, interference of pain with enjoyment of life and pain interference with general activity. Patients were also asked to respond to a two part Patient Self Evaluation (PSE) question: (i) a ranking on a scale of 1 to 7 for their perception of how much benefit they received ( $1=$ no benefit; $7=$ great deal of benefit and (ii) a write in response on the most important benefit (56\% indicated pain reduction). A parallel survey was submitted to providers. The PSE responses from patients and health care practitioners on perceived benefits and perceived negative effects were reported in free-text format. These were matched to the patient's assessment of benefit score and, according the study authors, these responses were a significant part of the study findings. Finally, respondents were asked also to report on symptoms associated with their pain: anxiety, appetite, depression, disturbed sleep, fatigue, nausea and vomiting. These are not captured by dispensaries. The reported symptom burden was high (apart from nausea and vomiting) with over $50 \%$ at baseline reporting one or more symptoms (sleep disturbance $91 \%$; depression $67 \%$ ). Overall, some 30 to $35 \%$ of respondents indicated a symptom improvement of $30 \%$ and retaining that for 4 months.

While the Minnesota study has provided a wealth of information on patient characteristics and the impact of botanical cannabis, it is only a single study. The claims presented for the impact of botanical cannabis should be evaluated in other jurisdictions. Even so, it would obviously be impossible to replicate the Minnesota model in treatment practice and it is unclear whether other state departments of 
health would be prepared to underwrite such studies. The respondent burden, especially for providers, would be significant, particularly when the range of conditions other than severe pain is the focus of botanical cannabis therapy (e.g., persistent muscle spasms, severe nausea, seizures, PTSD) and where many patients present with multiple conditions. Even so, the Minnesota survey demonstrates what is possible. The question is whether, as part of dispensary practice it is possible to utilize a registry or database design to capture response to therapy, where that response is captured in clinical terms as well as in the patient's own perception of the benefits of therapy, while at the same time minimizing respondent burden.

The passing of the Farm Bill 2018 gives added weight to the need for evidence to support the production and adoption of hemp-based CBD products. While hemp is a versatile agricultural crop, with multiple applications outside of THC-free CBD hemp oil, the evidence base is still limited for the conditions presented at dispensaries. Claims that THC-free CBD oils, for example, are more effective and with a lower likelihood of adverse events that THC/CBD combination oils are still an open question. Claims, therefore, to legislators, as part of a licensing application, that the proposed dispensary will focus on hemp-based CBD products should be treated with caution.

\section{FLOW OF INFORMATION}

At the outset, it should be clear that it is unlikely that the registry design would include, or need to include, inputs from health care practitioners. Apart from issues of coordinating health care provider response and the timing of that response over the course of treatment, both of which would impose a significant burden on providers, patient self-reporting on validated instruments would be a viable and even preferred option. In the case of chronic non-cancer pain, there are a range of validated PRO instruments that capture both the type of pain experience, distinguishing neuropathic from nociceptive pain, as well as evaluating the intensity of pain and functional status by body location. As shown both by existing evidence from RCTS and observational studies together with the detailed responses from the Minnesota study, response to botanical cannabis formulations can vary by type and location of pain. A dispensary should, therefore, be in a position to support claims that are specific to pain type and body location. The same argument applies to associated symptoms, in particular the experience of fatigue, anxiety, depression and sleep experience. Validated instruments are available for each of these conditions. Together with PROs for pain type and location, these instruments would provide a coherent and well-structured framework for tracking change over time and calibrating response to therapy by cannabis dosing and administrative form.

If claims are to be evaluated against cannabis dosing and administrative form, it is important that the registry tracks the THC:CBD ratios for products supplied at each dispensary visit. Although in their early stages, guidelines for the medicinal application of botanical cannabis emphasize, particularly for naïve consumers, the importance of starting at a low dose with minimum THC and titrating to an effective low THC/high CBD product ${ }^{7}$. The evidence for this is, however, limited. One concern from the Minnesota study, as noted above, is the proportion of patients on high THC products. Introducing the patient, particularly a naïve consumer, to a high THC dosing regimen may not only create risks for severe adverse events and cannabis dependence but obscure the clinical value of a more calculated and benign dosing regimen. Achieving target outcomes (e.g., a 30\% reduction in pain intensity within 3 months) with minimum psychoactive effects should be an integral part of the duty of care. It should be the responsibility of the dispensary to develop and adhere to dosing and formulation guidelines defined by administration route, reviewing patient response at regular dispensary visits. The question then becomes the willingness of the dispensary to host the registry (or contract hosting and administration to a third party) and utilize its own staff to coordinate with patients with data entry at each dispensary visit.

\section{QUESTIONS LEGISLATORS SHOULD ASK}

Given the feasibility of creating dispensary registries, the next question is to set the stage for registry data inputs and data outputs by establishing a minimum set of reporting criteria. If, for example, a proposed data collection protocol is proposed (as might be the case for future Minnesota studies of the contribution of botanical cannabis), what questions should a legislator ask to establish the status of the proposed registry? Or, these might be issues raised when adjudicating applications (typically well oversubscribed) for a limited set of state dispensary licenses. If there is competition between investors or multistate companies for limited state licenses, then it is presumably incumbent on legislators and agencies to set minimum standards for operational performance and quality in health care delivery. After all, in many states there is a continuing groundswell of opinion against legalizing access to medical marijuana. If this is to be countered, then legislators and registry operators need to demonstrate that they recognize a duty of care and are committed to achieving credible outcomes.

The following questions are proposed as the basis for medical marijuana dispensary licensing in the case of chronic noncancer pain. Similar questions could be raised in respect of the range of other approved treatment conditions:

- Has the dispensary adopted a registry design that allows patients with specific pain conditions to be monitored over the course of treatment?

- Does the dispensary have adequate product liability insurance to cover adverse outcomes and dispensary malpractice?

- Has this registry design and its data collection and reporting protocols been validated in other dispensaries? 
- Does the registry design require inputs from health care providers, patients and dispensary staff or only the patient and dispensary staff?

- Is the registry hosted and managed by the dispensary or is hosting and management contracted to a third party?

- Does the registry capture data from the patient at the initial dispensary visit and for all subsequent visits?

- Does the registry identify all disease and conditions for which medical marijuana has been approved in the state reported by the patient?

- Is the dispensary a separate entity from physician groups that advise patients on their eligibility for a medical marijuana card and assist in the approval process?

- Does the data capture from patients include conditions or disease states that are not approved for medical marijuana?

- Does the registry staff have training that allows a clinical evaluation at initial assessment or point of sale of botanical marijuana options given comorbid disease states and possible drug-drug interactions?

- Does the registry have access to qualified medical professional staff that allows at initial assessment or point of sale a clinical evaluation of botanical marijuana options given comorbid disease states and possible drug-drug interactions?

- Does the registry have guidelines for the dosing and administration of botanical cannabis which meet standards proposed for initial dosing, dose titration and the avoidance of psychoactive response?

- Does the registry report the dosing and administrative form of botanical cannabis at each dispensary visit?

- Are the registry clinical staffs equipped to assess response to therapy by pain type and location and advice on adjustments to the dosing and administration regimen?

- Does the registry capture at each dispensary visit prescription medication use including opioid formulation?

- Does the registry capture both clinical responses to therapy as well as the patient's perception of the response over baseline?

- Does the registry utilize validated instruments to measure response to therapy?

- Do the validated instruments capture both responses in terms of pain as well as functional status?

- Do the validated instruments capture response for fatigue, anxiety, depression and sleep experience?

- Is it possible to determine from the instruments minimum clinical improvement?

- Are summary reports provided to the patient and, with permission, the provider following each dispensary visit?
- Do the patient summary reports detail clinical improvement over base for pain intensity, functional status and associated conditions?

- Does the registry generate dispensary level reports for outcomes by target patient group?

\section{INVESTORS AND DISPENSARY RISK}

Without wishing to be unduly alarmist, the lack of a firm evidence base to support botanical cannabis dispensing recommendations should give pause. Consider the case of CBD. The 'conventional' wisdom, as noted above, is that the psychoactive THC should be avoided in favor of CBD. Indeed, there are companies producing hemp derived CBD oil as the preferred product. In the absence of evidence for the relative benefits and harms of various THC/CBD ratio combinations across the myriad conditions proposed or allowed for botanical cannabis, no such judgement should be made. We should be looking towards condition specific guidelines, taking account of the possibility of patients presenting with multiple allowed conditions or symptoms together with other comorbidities. In the case of glaucoma, for example, a recent mouse study claims that the dominant CBD effect may worsen glaucoma and increase eye pressure ${ }^{8}$. While it has been shown that THC is effective in lowering eye pressure, the study found that a THC/CBD combination effectively blocked the THC effect. In the absence of a firm evidence base, it is incumbent upon dispensaries, if they accept a duty of care, to monitor patients response to botanical cannabis in both THC/CBD combinations or these used separately (and other cannabinoids) to evaluate effectiveness.

In the absence of an audit trail which links condition presented to the consequent THC/CBD dosing regimens, dispensaries put themselves at risk. A situation that is confounded even more when registries fail to record condition or indeed fail to have condition specific guidelines in place to support staff in making recommendations and informing patients as to potential harms. The view appears to be that botanical marijuana is a 'benign' product with minimal downside risks. The fact is that cannabis is being supplied, to clients who are typically described as 'patients', in a retail environment where the focus is on sales. The only medical marijuana audit that occurs is to ensure they do not exceed legislated maximum consumption in a given period.

Certainly, companies offer legal marijuana dispensary insurance cover, to include product liability and, presumably, what may be described as 'dispensary malpractice'. The question is whether a claim will be met by the insurer? The risk is that without the ability to demonstrate that the dispensary is attempting to minimize product risk through monitoring patients, the dispensary may be denied a claim on the grounds that it was not demonstrating a reasonable 'duty of care'. Are dispensary counter staff, or even a physician $24 / 7$ phone/text backup in a position to advise and monitor patients who seek relief from intraocular eye pressure? While glaucoma patients 
my represent only a fraction of patients, the same arguments apply to chronic pain and the other conditions presented at dispensaries..

\section{CONCLUSIONS}

It was argued in the previous commentary that given the importance of feedback on the benefits and risks of botanical marijuana, state agencies may mandate data collection and reporting by the dispensary as a licensing condition if dispensary owners are reluctant to make such a commitment. In the absence of a registry it is difficult to see how dispensary owners could make believable claims for the clinical benefits of their dispensary services. Rather, they would fall back on their retail presentation in promoting cannabis strains and delivery options supported by targeted discounting, putting claims for clinical efficacy to one side.

Unless medical marijuana dispensaries, or at least a significant proportion of them, invest in a registry model to monitor and report patient outcomes with botanical cannabis, there will be a reluctance to accept cannabis as a contribution to the management of severe or chronic pain. Unfortunately, it is unclear at this point as to the willingness of dispensaries to commit resources to registry development. Whether this is a viable situation is a moot point. Certainly, with evidence from Colorado, the introduction of recreational marijuana has had a substantial impact on medical marijuana registration. It is not clear if this means that patients are now self-medicating or that a proportion of medical marijuana certificates were simply a cover for recreational marijuana. It may simply reflect pricing differentials and an unwillingness to shoulder administrative costs and processes for certification. Unfortunately, we will never know unless there are efforts to track patients who may be presenting with, for example, 'intractable' pain conditions. We will continue to operate in an information vacuum but with some, albeit limited, confidence in the 'effectiveness' of botanical cannabis.

The impact of a recreational marijuana option should not be understated. While it may increase the overall market for cannabis, with Colorado for example now having over 500 medical and recreational shop fronts, it may inhibit the medicinal use of cannabis by providers and patients due to the continued absence of robust outcomes data. Pursuit of the taxation benefits from botanical cannabis should not hide the fact that the ultimate goal is to improve patient health.

If we are to encourage dispensaries to support outcomes reporting and assessment, then the cost of implementation of a registry package should be reasonable. It is not the intention to replicate multimillion dollar RCTs or similar studies. The intention should be to support a pricing structure for registry introduction and long-term hosting, probably by a third party, that encourages dispensary and, through the dispensary, patient involvement. This may, as noted in the previous commentary result in a two-tier medical marijuana dispensary structure with participating dispensaries offering provider coordinated care management. Reducing barriers to the acceptance of the place of medical marijuana in care management, particularly for older patients presenting with comorbid conditions, would be a major objective. This may overcome the reluctance of many states to accept medical marijuana programs.

Even with states that have medical marijuana programs, the adoption of a registry option for dispensaries would indicate their acceptance of the need to support an enhanced evidence base for medical marijuana and an effective risk management strategy. Potential benefits in the duty of care and potentially improved outcomes for patients should be stressed. Beyond the enhancement of the presently fragmented evidence base, in the longer term a major benefit would be the utilization of observational monitoring of outcomes as inputs to the development and adoption of evidence-based, conditionspecific botanical cannabis guidelines. If so, then we may move towards a more effective and safer utilization of botanical cannabis as an integral part of pathways of care.

Conflict(s) of Interest: None declared 


\section{REFERENCES}

${ }^{1}$ Langley PC. Outcomes, registries and medical marijuana: Towards establishing dispensary monitoring and reporting standards. Inov Pharm. 2018;9(4): No. 7

${ }^{2}$ European Monitoring Centre for Drugs and Drug Addiction. Medical Use of cannabis and cannabinoids: Questions and answers for policy making. Publications Office of the European Union, Luxembourg. December 2018

${ }^{3}$ Andreae M, Carter G, Shaparin N et al. Inhaled cannabis for chronic neuropathic pain: a meta-analysis of individual patient data. $J$ Pain. 2015; 16: 1221-1232.

${ }^{4}$ Mucke M, Phillips T, Radbruch L et al. Cannabis-based medicines for chronic neuropathic pain in adults. Cochrane Database of Systematic Reviews. 2018; 3. CD012182.

${ }^{5}$ Stockings E, Campbell, G, Hall, W et al. Cannabis and cannabinoids for the treatment of people with chronic non-cancer pain conditions: a systematic review and meta-analysis of controlled and observational studies. Pain. 2018; 159:1932-1954

${ }^{6}$ Krebs, E. E., Lorenz, K. A., Bair, M. J., Damush, T. M., Wu, J., Sutherland, J. M., Asch S, Kroenke, K. Development and initial validation of the PEG, a three-item scale assessing pain intensity and interference. J Gen Int Med, 2009. 24(6), 733-738

${ }^{7}$ MacCallum CA, Russo EB. Practical considerations in medical cannabis administration and dosing. Eur J Int Med. 2018;49:12-19

${ }^{8}$ Miller S, Daily L, Leishman E et al. $\Delta^{9}$-Tetrahydrocannabinol and cannabidol differentially regulate intraocular pressure. Invest Ophthalmol Vis Set. 2018;59:5904-5911 